\title{
Estrategia participativa vivencial de educación musical en la calidad de la formación para estudiantes de educación inicial
}

\author{
Experiential participatory music education strategy in the quality of training \\ for early childhood students
}

\section{Estratégia participativa de educação musical participativa na qualidade do treinamento para estudantes de educação inicial}

Eloy Eladio Ayala Falcón Universidad Nacional Mayor de San Marcos, Perú eeayalaf@yahoo.com https://orcid.org/0000-0002-7592-5134

\section{Resumen}

El presente estudio evalúa la influencia de la estrategia participativa vivencial de educación musical en la calidad de la formación profesional de estudiantes de Educación Inicial de la Universidad Nacional Mayor de San Marcos. La investigación se enfoca en determinar la forma en que la estrategia participativa vivencial de educación musical contribuye a elevar los niveles de calidad de la formación profesional de los estudiantes de la referida carrera. El método de investigación fue cuasiexperimental. La población y muestra estuvo constituida por el total de alumnas matriculadas en tercer ciclo en la Universidad Alas Peruanas (grupo de control) y el total de alumnas matriculadas en un mismo ciclo de la Universidad Nacional Mayor de San Marcos (grupo de estudio). La prueba estadística aplicada fue Kolgomorov-Smirnov, ya que evalúa la dependencia de una variable cualitativa bimodal (estrategia A y B) con una variable cuantitativa discreta (notas de las alumnas). Se concluye que la aplicación de la estrategia participativa vivencial de 


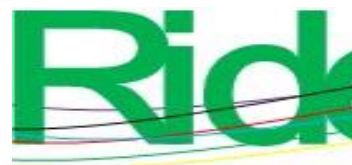

Revista Iberoamericana para la
Investigación y el Desarrollo Educativo
ISSN $2007-7467$

educación musical eleva la calidad de la formación profesional y humana de las estudiantes de Educción Inicial de la Universidad Nacional Mayor de San Marcos.

Palabras claves: calidad de la formación profesional, estrategia participativa vivencial, formación integral personal y humana.

\begin{abstract}
The present study evaluates the influence of the experiential participatory strategy of music education on the quality of the professional training of the initial education students of the National University of San Marcos. It consists of determining the experiential participatory strategy of music education contributes to raising the quality levels of the professional education of the students of initial education. The research method was quasi-experimental. The population and sample was constituted by the total of students enrolled in the third cycle in the Alas Peruanas University, control group, and total of students enrolled in the same cycle of the National University of San Marcos are study group. The applied statistical test was Kolgomorov-Smirnov since it evaluates the dependence of a bimodal qualitative variable (Strategy A and B) with a discrete quantitative variable, students' grades. Conclusion. The application of experiential participatory strategy of music education elevates the quality of professional and human formation of the initial students of the National University of San Marcos.
\end{abstract}

Keywords: quality of professional training, experimental participatory strategy, personal and human integral training.

\title{
Resumo
}

O presente estudo avalia a influência da estratégia experiencial da educação musical participativa na qualidade da formação profissional de estudantes de Educação Inicial da Universidade Nacional de San Marcos. A pesquisa se concentra em determinar de que maneira a estratégia participativa da educação musical experimental contribui para elevar os níveis de qualidade da formação profissional dos estudantes da carreira mencionada. $\mathrm{O}$ método de pesquisa foi quase experimental. A população e a amostra consistiram no número total de alunos matriculados no terceiro ciclo da Universidade Alas Peruanas (grupo controle) e no número total de estudantes matriculados no mesmo ciclo da 


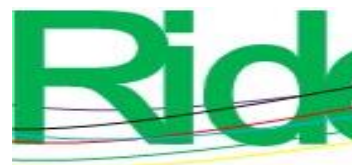

\section{Revista Iberoamericana para la Investigación y el Desarrollo Educativo ISSN $2007-7467$}

Universidade Nacional de San Marcos (grupo estudo). O teste estatístico aplicado foi Kolgomorov-Smirnov, uma vez que avalia a dependência de uma variável qualitativa bimodal (estratégias A e B) com uma variável quantitativa discreta (notas do aluno). Conclui-se que a aplicação da estratégia participativa de educação musical experiencial eleva a qualidade da formação profissional e humana dos estudantes de Educação Inicial da Universidade Nacional de San Marcos.

Palavras-chave: qualidade da formação profissional, estratégia participativa experiencial, treinamento integral pessoal e humano.

Fecha Recepción: Mayo 2019

Fecha Aceptación: Diciembre 2019

\section{Introducción}

Según Ayala (2012), en Perú existe la imperiosa necesidad de reformar y actualizar el sistema educativo. Esta afirmación del referido autor, por supuesto, involucra a todos, principalmente a los profesionales de educación que intentan elevar la calidad educativa y optimizar la formación del docente. Por esta razón, la mejora de la instrucción docente a nivel profesional y humano es fundamental para la sociedad actual.

Los diversos modelos pedagógicos ensayados en nuestro país, como la reforma educativa de la década de 1970, los quinquenios educativos en función de las políticas de los gobiernos de turno y el Proyecto Educativo Nacional al 2021 [PEN] (La educación que queremos para el Perú) presentan enfoques todavía parciales alejados del contexto actual. En tal sentido, resaltamos el objetivo estratégico cinco, acerca la formación docente: "Educación superior de calidad se convierte en factor favorable para el desarrollo y la competitividad nacional” (PEN, 2006). Sin embargo, como señala Ayala (2012), "a pesar de que la última reforma educativa expresada en el proyecto educativo nacional es importante para el sistema educativo, este enfoque no toma en cuenta a los actores principales que son los maestros y alumnos" (p. 127).

En tal sentido, el presente estudio demuestra que la calidad educativa tiene correlación con la formación integral de las docentes tanto en las áreas cognitivas como en las formativas, ya que estas son una unidad: "Es decir, vemos al ser humano como uno y a 


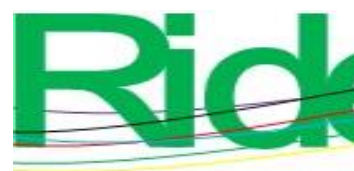

Revista Iberoamericana para la
Investigación y el Desarrollo Educativo
ISSN $2007-7467$

la vez pluridimensional, bien diverso como el cuerpo humano y a la vez plenamente integrado y articulado en una unidad” (Rincón, 2008, párr. 1).

Ahora bien, con base en los resultados encontrados, estamos convencidos de que el manejo de estrategias de enseñanza- aprendizaje adecuadas para el área y el nivel educativo tendrán resultados positivos, y que la interiorización de la estrategia participativa vivencial en la expresión creativa y apreciación musical mejorará la calidad profesional de la docente en el nivel inicial. Además, coincidimos con la declaración del representante del magisterio nacional acerca del tema que es materia de estudio, concedida a Palabra del MaestroRevista Pedagógica y Cultural de la Derrama Magisterial № 55 - abril 2011: "Necesitamos revisar el currículo de los Institutos y Universidades Nacionales y Privados por el cual se forman los profesionales en educación, requerimos una formación en servicio de calidad" (Villena, 2011, p. 5)

El sustento de la variable estrategia participativa vivencial toma relevancia en el pensamiento del filósofo Henri Bergson, quien explica "que una vivencia no es la idea de una cosa sino la experiencia" (Hirschberger, 1981, p. 380). En efecto, mientras que una idea es una representación, un concepto o un producto del intelecto, la vivencia es estar en presencia del objeto, vivirlo y explorarlo tal vez sin poder llegar a conocerlo nunca del todo, aunque pudiendo desarrollar solo algunas cuestiones desde algunas perspectivas. Al respecto, Bergson declara: "Todo ser es conciencia, pero no es conciencia kantiana entendida intelectualmente, sino conciencia como vida, vivencia, impulso, duración, libertad, creación, energía creadora" (Hirschberger, 1981, p. 380).

Para Bergson la filosofía es el ser como vida, y esta vida consiste en un continuo fluir sin interrupción; es un movimiento vital y la conciencia es equivalente a creación y libertad. Para una real formación musical hay que "hacer" música, para lo cual, según García (1891), necesitamos "tener" una vivencia. Este autor, citando un ejemplo de Bergson, explica lo siguiente:

Una persona puede estudiar minuciosamente el plano de París; estudiarlo muy bien, notar uno por uno los diferentes nombres de las calles, estudiar sus direcciones, luego puede estudiar los monumentos que hay en cada calle, puede estudiar los planos de esos monumentos, puede repasar las series de las fotografías del Museo de Louvre, una por una... puede llegar de esa manera a tener una idea regularmente clara, muy clara, clarísima, 


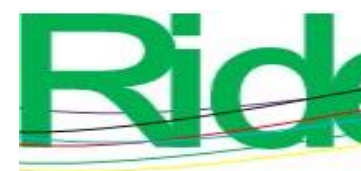

Revista Iberoamericana para la
Investigación y el Desarrollo Educativo
ISSN $2007-7467$

detalladísima de París. Esta idea podrá ir perfeccionándose cada vez más pero siempre será una mera idea. En cambio veinte minutos de paseo a pie por París es una vivencia. Entre esos veinte minutos de paseo a pie por una calle de París y la más larga y minuciosa colección de fotografías, hay un abismo. La una es una mera idea, una representación, un concepto, una elaboración mental; mientras que la otra es ponerse uno realmente en presencia del objeto, esto es: vivirlo, tenerlo propia y realmente en la vida ( $\mathrm{p}$. 2).

Esta interesante interpretación que realiza García de los términos hacer y tener nos permite esclarecer la propuesta de la estrategia participativa vivencial, así como profundizar en el estar presente. En concordancia con esta idea, consideramos importante para la participación vivencial en educación musical el ejercicio de la libertad y la comunicación asertiva entre las participantes para facilitar la relación con los demás y la vivencia directa con los sonidos mediante la entonación grupal de canciones infantiles y recreativas, acompañadas con los instrumentos musicales y/o con otros elementos sonoros.

Sobre la libertad en la educación, Montero (1989) plantea lo siguiente:

La educación será verdadera e integralmente humana si se ve en todo momento al que se educa como una inteligencia capaz de pensar por sí misma, una afectividad capaz de sentir, una motricidad capaz de manifestarse. Por ello educar es también preparar para el cambio. Preparar para el futuro querrá decir prepararse para la libertad desarrollando la propia libertad. El desarrollo de la propia libertad es una tarea de carácter vitalicio que afecta a quien educa y a quienes se educan. También de un modo general, educación equivale al desarrollo de la personalidad y desarrollarla es una tarea de libertad humana. Desde ambas perspectivas se llega a lo mismo: a la libertad y a la educación (p. 32).

Asimismo, la comunicación es impresindible para la convivencia armónica y la realización interpersonal e intrapersonal. Por ello, Blázquez (2006), en Emmanuel Mounier - grandes escritores contemporáneos, cita textualmente a Mounier: "La comunicación es quizá la experiencia fundamental de la persona. Ser hombre es vivir con, entre los hombres" (p. 154). Con esto, el referido autor nos explica el significado de la comunicación mounieriana como una apertura hacia el otro, en la que se produce un acto de descentrarse 


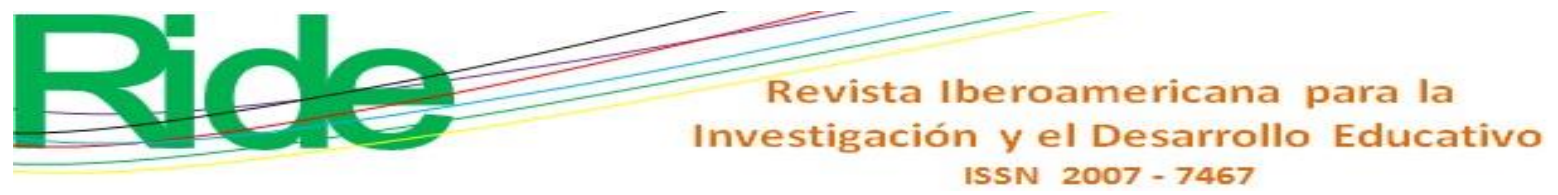

de sí mismo; es la capacidad de descubrirse en los otros, porque las otras personas no lo limitan, sino que lo hacen crecer.

Para Mounier los criterios fundamentales de la comunicación son salir de sí (el ser es cuando está con otros seres), comprender (ser para todos sin dejar de ser), asumir (no solo comapadecer, sino sufrir con el dolor, la pena y alegría), dar (la persona es generosidad y gratuidad) y ser fiel (fidelidad a la propia persona).

En esta misma línea, el destacado representante de la filosofía vitalista, Wilhelm Dilthey, en su obra Psicología de la estructura, acuña el concepto entender, según el cual la vida, presente en cada momento, penetra con su carácter peculiar y único brote nuevo de vida, sellándolo desde sí misma con su irrepetible individualidad. La manera como vivo yo ahora mis contenidos de representación depende del estado de conciencia de quien los recibe, y la manera como recibiré en mí y viviré mi futuro depende igualmente de mi estado de conciencia como base total desde la cual obro. Este estado de conciencia, entonces, es el mismo vivido ordinariamente como un determinado estado de sentimiento o como una deterninada actitud de voluntad (Hirschberger, 1981).

En la escuela activa, desde el comienzo y hasta los niveles superiores, uno de los principios esenciales tiene que ver con la vivencia como punto de partida para llegar a la teoría: "Vivir inconscientemente los fenómenos musicales, para tomar conciencia de ellos y así llegar a la vida consciente" (Willems, 2002, (p. 50).

En consecuencia, la educación musical como una experiencia vital y existencial es sustentada por la filosofía (como interpretación y valoración de la realidad), la epistemología (relación objetiva con la naturaleza: el aire y el sonido), la sociología (sentir la presencia y tener una convivencia armoniosa con los demás mediante las actividades colectivas que supone la música), la pedagogía (enfoques en función de los intereses y necesidades de los educandos) y la neurociencia (un sistema, una estructura de sensaciones neuronales ligadas al cerebro). Estas, según Picardo y Escobar (2002), son en la actualidad la base fundamental de la educación. En nuestra opinión, es el cimiento o la "infraestructura de la educación". 


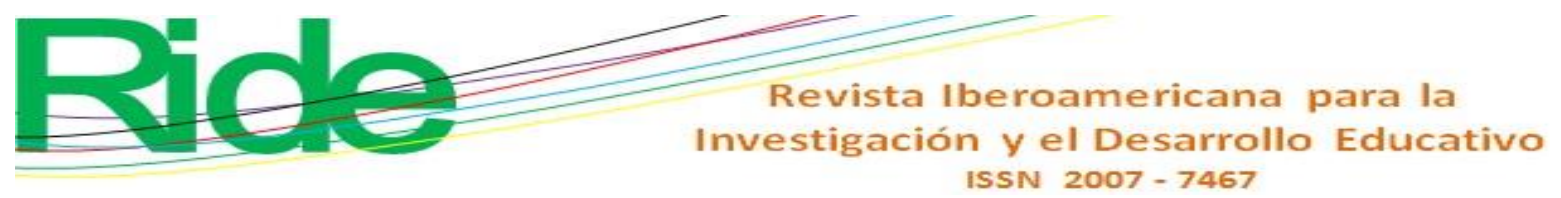

Fundamento teórico

La importancia de la educación musical y el arte en general en el nivel inicial se fundamenta en la necesidad de descubrir y desarrollar las potencialidades artísticas (entre ellas las musicales) de los niños y niñas como parte de su ser; por lo tanto, la música no debe ser entendida solo como una estrategia de recreación, sino también como una experiencia capaz de producir vivencias significativas. En palabras de Marco (2017), "la música es considerada un universal antropológico, esto es, un fenómeno que de una manera u otra se da en todas las culturas históricas o actuales" (p. 20). Así lo ha planteado el Ministerio de Educación (MINEDU) a través de la Dirección de Educación Inicial en el XIV Seminario Nacional de Programas de Atención no Escolarizado y Educación Inicial:

La música siempre estuvo íntimamente relacionada con el desarrollo de la humanidad, a través de sus diversas manifestaciones. Desde las épocas más primitivas, el ser humano incorporó la música a su existencia: lo acompañaba cuando adoraba a sus dioses, cuando danzaba, para aliviar sus pesares, en la guerra, en el trabajo, en la curación de los enfermos. También trató de reproducir, ya sea con su propia voz o fabricando instrumentos para golpear o con cuerdas para pulsar o frotar con un arco, los sonidos que escuchaba. Desde ese momento, la música surgió como un elemento indispensable en su vida" (MINEDU, 2011, p 2).

Asimismo, Valencia (2015), en El legado de Edgar Willems a la educación musical de hoy, cita a Willems acerca de la relación de la música con el ser humano:

Desde el momento cuando observé la música desde al ángulo de la educación, descubrí que la fuente de vida de los elementos musicales: sonido, ritmo, melodía armonía, improvisación, composición, no estaban en el conocimiento de la enseñanza académica, sino en el ser humano, en su múltiple naturaleza, dinámica, sensorial, afectiva, mental e ideal (p. 48).

Otro de los aspectos importantes para la educación musical en el nivel inicial es el estudio realizado acerca del desarrollo intrauterino del órgano auditivo, pues se ha demostrado que el oído es uno de los sentidos que se potencia antes que el resto, como lo explica Cabrelles (2006): 


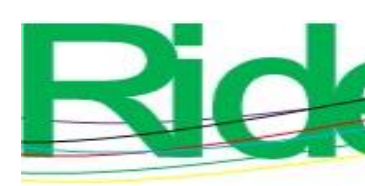

Revista Iberoamericana para la
Investigación y el Desarrollo Educativo
ISSN $2007-7467$

La aportación más importante del Dr. Tomatis fue descubrir que el feto oye sonidos en el vientre materno y además que la voz de la madre funciona como "cordón umbilical sónico" potenciando el desarrollo del bebé y constituyendo así una fuente primaria de estimulación. De esta forma estableció que dicho "cordón umbilical sónico" estaba constituido por el conjunto de sonidos percibidos por el feto durante su estancia en el útero materno y que éstos podían provenir de los ruidos corporales de la madre (masticar, eructar, tragar, ritmo respiratorio, ritmo cardíaco, sistema nervioso, etc.) y también de su voz (gritos, susurros, timbre de voz, etc.) (párr. 14).

Lozano y Lozano (2007) aporta una importante investigación acerca de los movimientos del corazón (sístole y diástole), los cuales se pueden emplear como didáctica para comprender el pulso. En tal sentido, el pulso fuerte es acento producido por sístole que determina el compás de una estructura musical y, en consecuencia, el compás del corazón es de dos tiempos: sístole y diástole. Por eso, los referidos autores afirman que el ser humano desde su gestación está en "movimiento". Su primer latido cardiaco, su frecuencia respiratoria y las pataditas en el vientre materno demuestran que el ritmo es parte de la vida:

El hombre es un ser de vibración, por tanto la música compuesta de vibración sonora incide directamente sobre el ser. La música es vida. El ser humano es la máxima expresión de la vida. Por lo que es posible que utilice la música como fin o como medio para armonizar todas sus dimensiones, tanto físicas como emocionales (Lozano y Lozano, 2007, p. 04)

A esta idea se puede agregar la opinión de Pino (2011):

La música arrastra a la acción y la interacción a través del ritmo. El ser humano es el único ser que puede sincronizar sus movimientos a través de la música, incluso siendo bebés, lo que contribuye a crear nexos sociales desde el corazón hasta el ritmo de los tambores (p. 49).

También una de las investigaciones más importantes que ha realizado la neurociencia tiene que ver con la relación de los estímulos sonoros con el cerebro. Esta disciplina ha demostrado que la música activa áreas del cerebro que son claves para el aprendizaje porque potencia y estimula las capacidades cognitivas, afectivas y motoras 


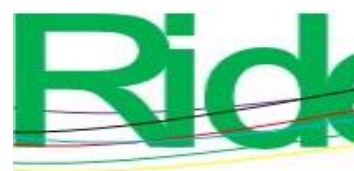

Revista Iberoamericana para la

Investigación y el Desarrollo Educativo

ISSN $2007-7467$

(Pino, 2011). Al respecto, Despins (1996) afirma que "el ritmo musical estimula los dos hemisferios cerebrales. El hemisferio derecho recibe el estímulo musical y el izquierdo interpreta, controla la ejecución. La música siempre será el mejor medio para desarrollar y acrecentar en forma adecuada este fenómeno cerebral" (p. 04). Esto explica la evidente relación que existe entre la música y el cerebro, la cual favorece a todos los seres humanos en proceso de desarrollo. Santiago (2009) lo analiza de esta manera:

La posibilidad de estimular el funcionamiento de los hemisferios del cerebro por medio de la actividad musical evidencia la necesidad de la educación artística y musical con la misma importancia de las otras disciplinas académicas. La enseñanza y aprendizaje de la música en particular y de todas las artes en general, fomentan un desarrollo integral de la mente, de la capacidades afectivas y de la sensibilidad del estudiante (p. 52).

En consecuencia, la educación musical en la formación de las docentes de educación inicial es imprescindible porque en ella "la integración de los tres aprendizajes fundamentales para el desarrollo del ser humano: hacer, conocer y convivir, tienen en la música un aliado fundamental que va unido al proceso de desarrollo y aprendizaje de niños y niñas (Rivas, 2005, p. 4)

Por nuestra parte, siempre estamos motivando a las alumnas en los espacios académicos o en otros eventos porque valoramos la importancia de la educación musical en la formación integral. Al respecto, coincidimos con la siguiente frase de Ayala (2017): "Una docente de inicial que ama su vocación, canta, baila y toca algún instrumento será una docente competente y profesional exitosa" (p. 29). Este autor también indica que el pensamiento es bien recibido e interiorizado por cada una de las docentes, quienes manifiestan una actitud positiva frente a su carrera profesional, desarrollo y formación personal.

\section{Metodología de la investigación}

El estudio se centró en el área de educación, específicamente en el manejo y aplicación de la estrategia participativa vivencial de educación musical en la calidad de la formación profesional y humana de las futuras docentes, estudiantes de la especialidad de Educación Inicial de la Escuela Académico Profesional de la Facultad de Educación de la Universidad Nacional Mayor de San Marcos. 


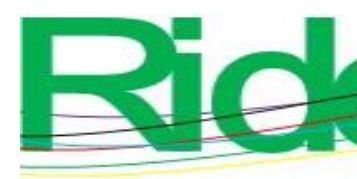

Revista Iberoamericana para la

Investigación y el Desarrollo Educativo

ISSN $2007-7467$

\section{Tipo de investigación}

El diseño del presente estudio corresponde al tipo de investigación cuasiexperimental, pues "se trabaja con dos grupos que son casi iguales o con grupos supuestamente iguales pero el investigador no los ha formado sino que los encuentra ya formados" (Mejía, 2017, p. 64).

Aunado a esto, como señalan Hernández, Fernández y Baptista (2014), "los diseños cuasi-experimentales tienen el mismo propósito que los estudios experimentales: probar la existencia de una relación causal entre dos o más variables (p. 151).

En la presente investigación hemos encontrado a dos grupos ya formados por estudiantes de la misma especialidad que cursaban el mismo año académico con planes de estudios similares. El grupo de control estuvo conformado por las alumnas de Educación Inicial (semestres I y II, año 2017) de la Universidad Alas Peruanas (UAP), mientras que el grupo de estudio estuvo constituido por las alumnas de Educación Inicial (semestres I y II, año 2017) de la Universidad Nacional Mayor de San Marcos (UNMSM).

\section{Población de estudio}

La población de una investigación es definida por Mejía (2017) de la siguiente manera:

Una población es la totalidad de sujetos o elementos que tienen características comunes. En otras palabras, una población es la totalidad de los miembros de la unidad de análisis. El concepto de población equivale al concepto conjunto y éste es delimitado por el investigador según los criterios que considere pertinentes (...). Es muy importante que el investigador defina claramente la población porque como se ha visto, de esta definición depende el número de los elementos del conjunto (p. 201).

Tomando en cuenta esta observación, la población estuvo constituida por el total de alumnas matriculadas en los semestres I y II, año 2017, en una sola sección del mismo año de la especialidad de Educación Inicial de la Facultad de Educación de la Universidad Nacional Mayor de San Marcos y por el total de estudiantes de la Facultad de Ciencias Empresariales y Educación de la Universidad Alas Peruanas.

La población estudiada tuvo características comunes: estaban matriculadas y asistían regularmente a los cursos del respectivo año académico y de la especialidad. Esta 


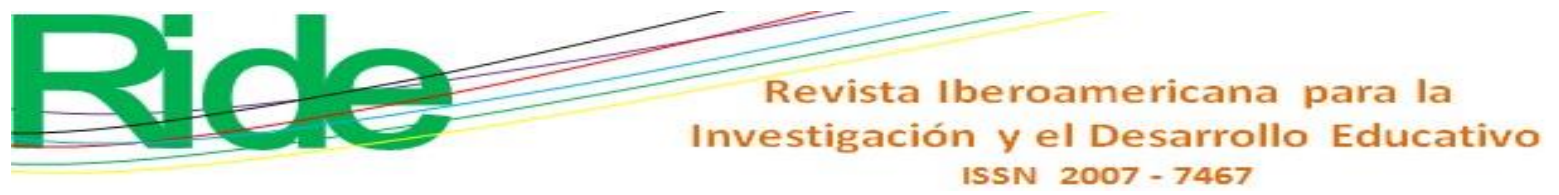

situación facilitó bastante la tarea de recolección, interpretación y análisis de los datos. En síntesis, los grupos quedaron conformados de la siguiente manera:

Grupo de control: Semestre 2017-I = 42; semestre 2017-II = 14 .

Grupo de estudio: Semestre 2017-I = 30; semestre 2017-II = 32 .

Total: Grupo de control 56 alumnas y grupo de estudio 62 alumnas.

\section{Muestra}

Para determinar la muestra se consideró la recomendación de Mejía (2017): "No existe un criterio definido acerca del tamaño recomendable que debe tener la muestra. La experiencia del investigador, los recursos que dispone o las facilidades técnicas que se le presentan, serán los determinantes para fijar el tamaño de la muestra. Sin embargo, desde la etapa de planificación de la investigación se debe fijar el tamaño de la muestra" (p. 206).

En definitiva, se trabajó con el total de alumnas matriculadas en el segundo año de la especialidad de Educación Inicial.

Grupo de control: Total semestres I y II $=56$.

Grupo de estudio: Total semestres I y II $=62$.

\section{Unidad de análisis}

Estuvo conformada por cada uno de los integrantes del grupo de alumnas de la especialidad de Educación Inicial con características propias y comunes que conforman un todo. En concreto, se determinó como unidad de análisis a las alumnas del segundo año, semestres I y II, año 2017, de la especialidad de Educación Inicial de la Escuela Académico Profesional de Educación de la Facultad de Educación de la Universidad Nacional Mayor de San Marcos, mientras que como grupo de estudio se seleccionó a una sección por semestre durante el desarrollo de las asignaturas Desarrollo de educación musical infantil (semestre I) y Didáctica para el desarrollo de educación musical infantil (semestre II), los mismos grupos como efecto o resultado de estudio al final del desarrollo de la asignatura, expresados en el promedio de notas finales de 0 a 20 del año académico 2017. 


\section{Diseño gráfico de la investigación}

\section{Diagrama del diseño}

$$
\text { Grupo que lleva la variable }=\frac{O_{1}}{O_{2}}=\frac{X}{Z}
$$

Tabla 1. Grupos de estudio y control (Ñaupas, Mejía, Novoa y Villagómez, 2011)

\begin{tabular}{|c|c|c|c|}
\hline Grupo & Preprueba & V. Independiente & Posprueba \\
\hline E & & $\mathrm{x}$ & $\mathrm{O}_{1}$ \\
\hline $\mathrm{C}$ & & - & $\mathrm{O}_{2}$ \\
\hline
\end{tabular}

Fuente: Elaboración propia

Donde:

E es el grupo de estudios.

C es el grupo de control.

Diseño cuasiexperimental solamente con posprueba.

\section{Resultados}

\section{Presentación, análisis e interpretación de los datos}

En la planificación del trabajo empírico se ha considerado más que una técnica de observación el análisis de resultados de dos grupos ya formados; por lo tanto, su validez interna fue pequeña, ya que no hubo control sobre las variables extrañas. Hemos trabajado en el campo de dos situaciones reales (un grupo de control y otro de estudio) con la finalidad de comparar las puntuaciones finales por la influencia de manejo de estrategias metodológicas diferentes.

Tabla 2. Número de alumnas de los grupos de control y de estudio (actas de matrícula UAP y UNMSM, 2017)

\begin{tabular}{|l|c|c|c|}
\hline \multicolumn{1}{|c|}{ GRUPOS } & SEMESTRE 2017-1 & SEMESTRE 2017-2 & TOTAL \\
\hline Control - UAP & 42 & 14 & 56 \\
\hline Estudio - UNMSM & 30 & 32 & 62 \\
\hline
\end{tabular}

Fuente: Elaboración propia 


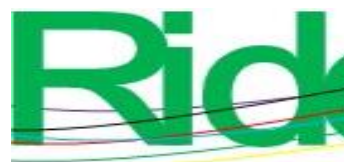

Revista Iberoamericana para la Investigación y el Desarrollo Educativo

ISSN $2007-7467$

Tabla 3. Promedio de notas de los grupos de control y de estudio (actas de evaluación final

UAP y UNMSM, 2017)

\begin{tabular}{|l|c|c|}
\hline & Control & Estrategia \\
\hline Promedio & 14 & 17 \\
\hline Máxima & 18 & 19 \\
\hline Mínima & 7 & 15 \\
\hline
\end{tabular}

Fuente: Elaboración propia

Figura 1. Histograma (resultado de promedios de notas, 2017)

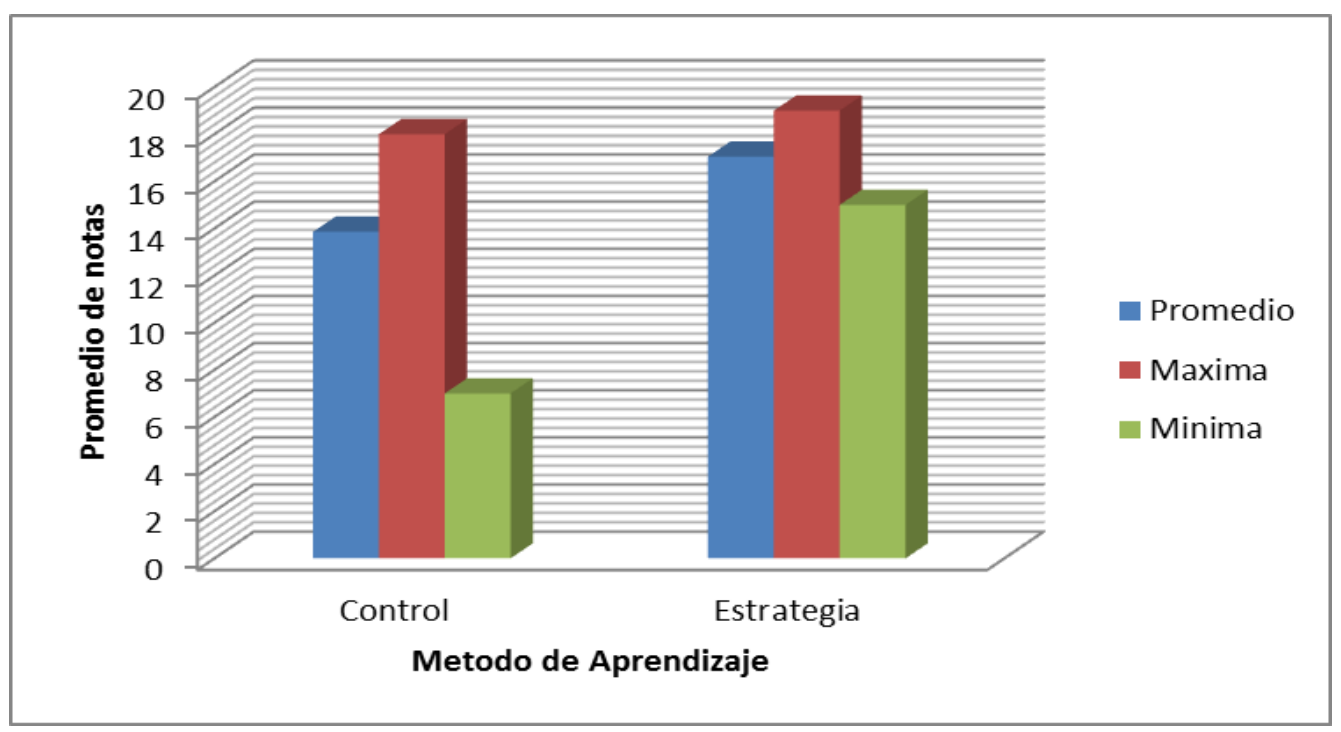

Fuente: Elaboración propia

\section{Análisis estadístico}

Se procuró demostrar de manera estadística si las notas obtenidas por los alumnos dependían o no del método pedagógico utilizado. Por lo tanto, la prueba estadística elegida para este caso fue Kolgomorov-Smirnov, ya que se evaluó la dependencia de una variable cualitativa bimodal (estrategia A y B) con una variable cuantitativa discreta (notas del alumnado) (Daniel, 2004).

La prueba estadística Kolgomorov-Smirnov es no paramétrica, ya que los datos no siguen la distribución normal. Los datos y la información del estudio fueron estudiados utilizando el paquete estadístico STATA12. Para la prueba de dependencia KolgomorovSmirnov las hipótesis fueron: 
- $\mathrm{H}_{\mathrm{O}}=$ No hay dependencia $(\mathrm{A}=\mathrm{B})$

- $\mathrm{H}_{\mathrm{a}}=$ Hay dependencia $\quad(\mathrm{A} \neq \mathrm{B})$

Tabla 4. Paquete estadístico STATA 12 - Kolgomorov-Smirnov ( $\mathrm{p}<0.05)$

\begin{tabular}{|llll|}
\hline Smaller group & D & P-value & Corrected \\
\hline $0:$ & 0.7535 & 0.000 & \\
$1:$ & 0.0000 & 1.000 & \\
Combined K-S: & 0.7535 & 0.000 & 0.000 \\
& & \\
Note: ties exist in combined dataset; & & \\
There are 11 unique values out of 118 observations & \\
\hline
\end{tabular}

Fuente: Elaboración propia

Utilizando el paquete estadístico STATA 12, el valor de $\mathrm{p}=0.000$. Por lo tanto, se rechaza la hipótesis nula y se acepta la alterna. Finalmente, se concluye que hay dependencia entre la estrategia utilizada y las notas del alumnado.

\section{Análisis y discusión}

Asumiendo que toda investigación es imperfecta, en nuestro caso por la complejidad y amplitud del tema, hemos puesto todo nuestro interés en un aspecto: "estrategia participativa vivencial", para lo cual se ha considerado una serie de acciones integradas y organizadas "que los alumnos deben realizar para alcanzar vivencias vinculadas a la vida, a su desarrollo físico y a aspectos no veritacionales de la cultura, tales como las actividades artísticas, religiosas, cívicas y morales entre otras de valor formativo" (Peñaloza, 2005, p. 387).

Si bien es cierto que el tema tiene mucha importancia para la educación, Willems (2002) en su preocupación por unir los elementos fundamentales de la música con los de la naturaleza humana, reconsidera a la luz de la nueva mentalidad científica el concepto de educación musical defendido por Platón y los pitagóricos, según el cual la música — como formadora del alma - contribuye a una mejor armonía del hombre consigo mismo, con la naturaleza y con el cosmos. 


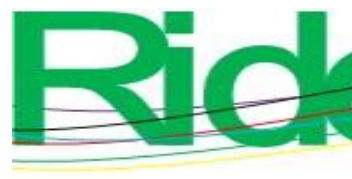

Revista Iberoamericana para la

Investigación y el Desarrollo Educativo

ISSN $2007-7467$

La metodología elegida fue adecuada y pertinente para una investigación cualitativa-cuantitativa, pero tiene limitaciones en la medida en que los resultados del estudio se han realizado con base en los hallazgos de las notas de un grupo pequeño de control y otro de estudio.

Aun así, los datos demuestran que se han cumplido los objetivos propuestos, es decir, determinar si la estrategia participativa vivencial de expresión musical creativa contribuye a elevar los niveles de calidad de la formación profesional y humana de las estudiantes de Educación Inicial de la Escuela Académico Profesional de la Facultad de Educación de la Universidad Nacional Mayor de San Marcos.

Asimismo, manifestamos que el presente estudio de "estrategia participativa vivencial" de expresión creativa y apreciación crítica para la educación musical en el nivel inicial abre las puertas para las futuras investigaciones de otros temas en el contexto de la problemática de la música en educación.

\section{Conclusiones}

La estrategia participativa vivencial de educación musical es un factor que contribuye a elevar los niveles de calidad en la formación profesional de estudiantes de Educación Inicial de la Facultad de Educación de la Universidad Nacional Mayor de San Marcos, según las siguientes dimensiones:

En la dimensión profesional, la educación vivencial de los elementos básicos de la música es indispensable para la carrera y la formación profesional de calidad de las docentes de educación inicial.

En la dimensión personal, las expresiones musicales individuales y grupales desarrollan sus posibilidades de realización personal, seguridad, confianza en sí mismo y su identidad.

En la dimensión humana, la educación musical crea personas con iniciativa, respeto a la dignidad humana, autodeterminación y un sentimiento de solidaridad con los semejantes sin dejar de desarrollar la individualidad.

Finalmente, se debe indicar que la capacitación de las futuras pedagogas es responsabilidad del Ministerio de Educación, de las universidades y de los institutos pedagógicos, los cuales tienen la tarea de formar a las docentes en la especialidad de 


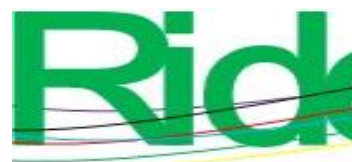

Revista Iberoamericana para la

Investigación y el Desarrollo Educativo

ISSN $2007-7467$

educación inicial tomando en cuenta el ejercicio eficaz de la docencia, el desarrollo y la transformación de la persona y la generación de ideas creadoras, respuestas inéditas y originales a los problemas concretas del contexto social.

\section{Referencias}

Ayala, E. (2012). Formación docente bajo el paradigma humanístico. (Tesis de maestría). Universidad Nacional Mayor de San Marcos. Lima, Perú.

Ayala, E. (2017). Estrategia participativa vivencia de educación musical y calidad en la formación profesional de estudiantes de educación inicial de la Facultad de Educación de la Universidad Nacional Mayor de San Marcos. (Tesis doctoral). Universidad Nacional Mayor de San Marcos. Lima, Perú.

Blázquez, F. (2006). Emmanuel Mounier - Grandes escritores contemporáneos. Madrid: EPESA.

Cabrelles, M. (2006). Los sonidos de nuestro cuerpo: los ruidos biológicos. Valladolid, España. Biblioteca Virtual Miguel de Cervantes.

Daniel, W. (2004). Bioestadística. Bases para el análisis de las ciencias de la salud (4. ${ }^{\mathrm{a}}$ ed.). México, D. F.: Balderas 95.

Despins, J. P. (1996). La música y el cerebro. Ciencias/Cognitivas. Editorial GEDISA.

García, M. (1981). Lecciones preliminares de filosofía (23. a ed.). Buenos Aires, Argentina: Editorial Losada S. A.

Hernández, R., Fernández, C y Baptista, P. (2014). Metodología de la investigación (6. ${ }^{\mathrm{a}}$ ed.). México, D. F.: Mc Graw Hill Education.

Hirschberger, J. (1981). Historia de la filosofía (tomo II). Barcelona, España: Editorial Herder.

Lozano, L. y Lozano, A. (2007). La influencia de la música en el aprendizaje. Memorias del IX Congreso Nacional de Investigación Educativa. Mérida, México.

Marco, T. (2017). Escuchar música de los XX y XXI. Bilbao, España. Fundación BBVA. Edición y Producción: Moonbook.

Mejía, E. (2017). La investigación científica en educación. Lima, Perú: Ediciones APEEM. 


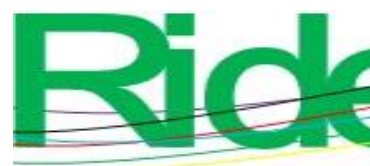

Revista Iberoamericana para la
Investigación y el Desarrollo Educativo
ISSN $2007-7467$

Ministerio de Educación [MINEDU] (2011). Importancia de la música en el desarrollo infantil. XIV Seminario Nacional de programas de atención no escolarizado y educación inicial. Dirección de Educación Inicial. Trujillo, La Libertad, Perú.

Montero, A. (1989). La libertad en la educación. Recuperado de http://institucional.us.es/revistas/cuestiones/6_7/art_3.pdf.

Ñaupas, H., Mejía, E., Novoa, E. y Villagómez, A. (2011). Metodología de la investigación científica y asesoramiento de tesis (2. ${ }^{\mathrm{a}}$ ed.). Lima, Perú: Centro de Producción Editorial e Imprenta de la Universidad Nacional Mayor de San Marcos. CEPREDÍM.

Peñaloza, W. (2005). El currículo integral. Lima, Perú: Ediciones UNMSM.

Picardo, O. y Escobar, J. (2002). Educación y sociedad del conocimiento: introducción a la filosofía del aprendizaje (pensar y enseñar a aprender a partir de la historia). San José de Costa Rica: Imprenta Obando S. A.

Pino, M. (2011). Reflexiones sobre música y neurociencia. Revista de Medicina y Humanidades. Vol. III N³, 2011.

Proyecto Educativo Nacional al 2021 [PEN] (2006). La educación que queremos para el Perú. Concejo Nacional de Educación. Lima, Perú. Edición con el Apoyo de USAID.

Rincón, L. (2008). El perfil del estudiante que pretendemos formar en una institución educativa ignaciana. Presidente de la Federación Latinoamericana de Colegios de la Compañía de Jesús (FLACSI) y de la Asociación de Colegios Jesuitas de Colombia (ACODESI).

Rivas, E. (2005). Aspectos fundamentales de la música en la educación inicial. Caracas, Venezuela: Ministerio de Educación y Deporte.

Santiago, E. (2009). Música como estrategia educativa en el proceso enseñanza aprendizaje para el aprovechamiento académico de los estudiante a nivel elemental en la región educativa de Bayamón (tesis de maestría). Río Piedras, Puerto Rico. Maestría en Administración y Supervisión Educativa. Universidad Metropolitana.

Valencia, G. (2015). El legado de Edgar Willems a la educación musical de hoy. Herencia de Edgar Willems pedagogo del sigo XX, a la Willems pedagogía musical del siglo XXI. Ricercare, (4), 46-52. 


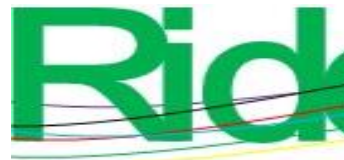

Revista Iberoamericana para la Investigación y el Desarrollo Educativo ISSN $2007-7467$

Villena, H. (2011). Proyecto Educativo Nacional. (Entrevista al Secretario General del CEN del SUTEP 2009-2011). Palabra de Maestro. Revista Pedagógica y Cultural Derrama Magisterial. № 55, Abril 2011. Lima Perú

Willems, E. (2002). El valor humano de la educación musical. Barcelona, España: Editorial Paidós 